\title{
Médecine de Catastrophe
}

\author{
Benjamin Davido
}

$\mathrm{M}$ EME SI LA FRANCE est le pays de Louis Pasteur, les Français ont longtemps été réticents à se faire vacciner. Dans les années 1990, le vaccin contre l'hépatite $B$ a été soupçonné de provoquer la sclérose en plaques'. Si le soupçon s'est avéré infondé, l'inquiétude qu'il a provoquée s'est révélée inflexible. Il n'est pas surprenant qu'à l'heure du COVID-19, les Français soient restés méfiants. En décembre 2020, quelque quarante pour cent du public français étaient prêts à accepter la vaccination ; fin janvier 2021, soixante pour cent ${ }^{2}$. Le déploiement du vaccin à ARNm de Pfizer-BioNtech s'est fait, à un rythme glacial : 1,6 million de doses étaient disponibles en France le ler février 2021. C'était un mois entier après le début de la campagne de vaccination, qui a commencé officiellement le 27 décembre 2020. Les médecins généralistes et les pharmaciens sont traditionnellement autorisés à administrer des vaccins, mais les vaccins à ARNm ; Pfizer et Moderna ; nécessitent une réfrigération profonde par supercongélateur, et ni les médecins généralistes ni les pharmaciens n'étaient en mesure de la fournir.

Il fallait un vaccin plus pratique.

C'est là qu'intervient AstraZeneca.

Nouvelle technologie de vecteur viral à partir de virus vivant, et on l'espère, fiable, le vaccin AstraZeneca ne nécessite lui pas de réfrigération profonde. S'il est stocké entre deux et huit degrés Celsius, il peut se conserver pendant au moins six mois ${ }^{3}$. En comparaison, le vaccin Pfizer peut être conservé jusqu'à trente jours, mais uniquement s'il est conservé à moins soixante-dix degrés Celsius, à plus ou moins dix degrés, et réapprovisionné en milieu réfrigéré tous les cinq jours. Avant d'être utilisé, il doit être décongelé, après quoi il peut être conservé pendant cinq jours, mais seulement s'il est stocké entre deux et huit degrés Celsius. Chaque flacon de Pfizer contient une quantité suffisante pour six à sept doses, qui doivent être utilisées dans les six heures, contre deux doses dans quarante-huit heures pour AstraZeneca ${ }^{4}$.

Le 29 janvier 2021, l'Agence européenne des médicaments (EMA), une agence de l'Union européenne qui évalue et supervise les médicaments, a donné son feu vert au vaccin d'AstraZeneca ${ }^{5}$. Le jour même de son approbation, le président français Emmanuel Macron s'est interrogé publiquement sur la nécessité de restreindre le vaccin d'AstraZeneca aux moins de soixante-cinq ans, le qualifiant de «quasi-inefficace » pour toute personne dépassant ce groupe d'âge ${ }^{6}$. "Ce que je peux vous dire officiellement, a-t-il fait remarquer, c'est que les premiers retours que nous avons ne sont pas aujourd'hui encourageants pour les plus de 60-65 ans sur AstraZeneca ».

Deux jours plus tard, le 2 février 2021, la Haute Autorité de Santé (HAS) recommande le vaccin AstraZeneca à « tous les citoyens » et à "l'ensemble des professionnels du secteur de la santé et du médico-social de moins de 65 ans $»^{7}$. Pourtant, le même jour, la HAS a publié un communiqué de presse dans lequel AstraZeneca était recommandé aux jeunes et aux personnes en bonne santé, mais pas aux personnes âgées, notant que « [1]es données actuelles ne [permettent] pas d'apprécier le niveau d'efficacité que ce vaccin procure chez les personnes de plus de 65 ans $»^{8}$. L'explication donnée à ces doutes était un « faible effectif de participants âgés de 65 ans et plus dans les essais ».

En l'espace de cinq jours, deux agences ; l'une européenne, l'autre française ; et le président français ont présenté des évaluations différentes de l'efficacité et des limites potentielles du vaccin d'AstraZeneca. Le résultat inévitable de ce message contradictoire des autorités a semé la confusion du public concernant ce vaccin.

C'est dans ce contexte incertain que les vaccinations d'AstraZeneca ont commencé. Il s'est avéré que le public français n'a pas eu à attendre longtemps pour que d'autres évaluations contradictoires apparaissent. Le 11 février 2021, l'ANSM (Agence nationale de sécurité du médicament et des produits de santé) constate que pour 10000 personnes injectées, environ 150 d'entre elles présentent des symptômes grippaux'. Le rapport de l'ANSM a créé une énorme inquiétude, notamment chez les infirmières : « [AstraZeneca], c'est un vaccin correct pour l'ensemble de la population générale », a observé Thierry Amouroux, porte-parole du Syndicat national des infirmières et infirmiers de France, «Mais par contre, pour une population aussi exposée que les soignants, c'est parmi les trois vaccins autorisés le moins efficace $»^{10}$.

Un mois plus tard, les nouvelles ne vont pas être plus rassurantes. Le 16 mars 2021, l'EMA a publié un communiqué 
de presse concernant un lien possible entre le vaccin d'AstraZeneca et des épisodes de thrombose ${ }^{11}$. Les symptômes de la grippe sont une chose, la thrombose en est une autre. Un caillot de sang se déplaçant vers le cerveau, le cœur ou les poumons qui pourrait bien s'avérer fatal. Néanmoins, l'EMA reste persuadée que «l'efficacité prouvée du vaccin dans la prévention des hospitalisations et des décès dus au COVID-19 l'emporte sur la probabilité extrêmement faible de développer [des caillots sanguins] ». Pour mettre cela en perspective, un article publié par The BMJ le 11 mars a noté que seulement « 30 cas d'événements thromboemboliques avaient été signalés parmi les cinq millions de personnes ayant reçu le vaccin d'AstraZeneca dans l'Espace économique européen $»^{12}$.

Compte tenu de l'avis initial de l'EMA, la France a choisi de ne pas suspendre immédiatement la campagne de vaccination d'AstraZeneca. En Allemagne, les vaccinations AstraZeneca avaient déjà été suspendues le 15 mars par mesure de précaution, sur les conseils de l'Institut Paul Ehrlich, l'autorité du pays en matière de vaccins ${ }^{13}$. Le véritable choc est survenu deux jours plus tard, le 17 mars, lorsque l'Élysée est revenu sur sa décision de maintenir le vaccin AstraZeneca, une volte-face qui n'a rien fait pour rassurer le public français. S'exprimant à titre confidentiel, une source de l'administration de M. Macron a admis que la décision antérieure de Berlin avait eu un «impact psychologique » sur le gouvernement français ${ }^{14}$.

L'EMA a d'abord semblé ne pas savoir à quels principes ses États membres faisaient appel pour suspendre le vaccin. Le 18 mars, la directrice exécutive de l'EMA, Emer Cooke, a répondu à une question concernant la possibilité d' « harmoniser l'application du principe de précaution », question qu'elle a repoussée de manière diplomatique, répondant qu' "il y a beaucoup de choses que nous devons faire pour nous assurer que tout le monde dispose des mêmes informations sur les avantages et les risques $»^{15}$. L'ambassadeur de l'UE au Royaume-Uni, João Vale de Almeida, avait d'autres idées. «Quand il y a des doutes, le principe de précaution prévaut », a-t-il fait remarquer le 16 mars $_{16}$. Et il n'était pas le seul. « En vertu du principe de précaution et dans l'attente d'informations complémentaires », le médecin en chef adjoint de l'Irlande, le Dr Ronan Glynn, a annoncé que « le NIAC (National Immunisation Advisory Committee) avait recommandé le report temporaire du programme de vaccination COVID-19 d'AstraZeneca en Irlande $»^{17}$.

Le principe de précaution a été formellement adopté dans le traité de Maastricht de 1992. En substance, cet article permet à l'UE de prendre des mesures préventives en cas de risque ; plus précisément, « lorsqu'un phénomène, un produit ou un procédé peut avoir des effets potentiellement dangereux, identifiés par une évaluation scientifique et objective $»^{18}$. Le philosophe suédois Per Sandin a défini le principe de précaution comme suit : "s'il existe une menace, qui est incertaine, alors une certaine forme d'ac- tion est obligatoire $»^{19}$. L'article est suffisamment vague pour permettre à l'UE de prendre des mesures même lorsque l'évaluation scientifique "ne permet pas de déterminer le risque avec suffisamment de certitude », comme l'UE elle-même l'a notée ${ }^{20}$. Dans leur petit livre sur le principe de précaution, Gary Marchant et Kenneth Mossman soulignent que

le traité lui-même ne définit pas ou n'articule pas autrement les exigences du principe de précaution, et aucune explication ou définition officielle du principe de précaution n'a été fournie au cours du processus de son incorporation dans le traité européen. Il a donc été laissé aux institutions communautaires le soin de définir et d'appliquer le principe de précaution ${ }^{21}$.

En 2000, la Commission européenne a publié un communiqué qui visait à

établir un accord sur les facteurs qui déclenchent le recours au principe de précaution et sur la place qu'occupe ce dernier dans le cadre de la prise de décision, ainsi qu’à énoncer des orientations pour l'application de ce principe, fondées sur des principes raisonnés et cohérents ${ }^{22}$.

Le document comprenait une série de principes généraux pour l'application du principe de précaution. Parmi ceux-ci figurait une analyse coûts-avantages qui impliquait

établir une comparaison entre le coût global pour la Communauté de l'action envisagée et de l'absence d'action, tant à court qu'à long terme. Il ne s'agit pas d'une simple analyse de rentabilité économique : sa portée est beaucoup plus vaste et inclut des considérations d'ordre non-économique, telles que l'efficacité d'options possibles et leur acceptabilité par la population. Dans la mise en oeuvre d'un tel examen, il faudrait tenir compte du principe général et de la jurisprudence de la Cour qui donnent la priorité à la protection de la santé par rapport aux considérations économiques ${ }^{23}$.

Cela signifie que les avantages et les inconvénients de toute décision prise sur la base du principe de précaution doivent être soigneusement pesés avant toute action. Dans le cas du vaccin d'AstraZeneca, l'intervention en France signifiait l'arrêt d'une campagne de vaccination déjà impopulaire et un nombre extraordinaire de vaccins gaspillés, alors que l'inaction aurait signifié la poursuite du déploiement d'un vaccin qui s'était déjà avéré efficace pour aider à freiner la spirale des cas de COVID au Royaume-Uni ${ }^{24}$. La décision n'a pas dû être facile à prendre. Hélas, si l'UE avait été contrainte de prendre une décision, peut-être aurait-elle été plus facile. Le scénario qui s'est déroulé, dans lequel chaque pays européen a choisi 
de décider seul, était imprévisible et a inévitablement créé un doute généralisé parmi les populations du bloc. Ce fut d'abord le choix du Danemark, puis de l'Islande, puis de la Norvège. La crise a touché toute l'Europe de la même manière et chaque membre de l'UE a appliqué le principe de précaution de la même façon.

Avant même que les inquiétudes concernant un éventuel lien avec les caillots sanguins ne commencent à apparaître à la mi-mars 2021, le vaccin d'AstraZeneca avait déjà été pointé du doigt en France. Début mars, un syndicat d'infirmières a exprimé des doutes sur le fait qu'AstraZeneca était le mieux adapté aux personnes présentant un risque élevé d'exposition au virus ${ }^{25}$. À l'époque, le gouvernement a déclaré qu'il n'y avait aucune raison de douter et a insisté pour que, malgré leurs inquiétudes, les travailleurs de la santé soient rapidement vaccinés avec le vaccin AstraZeneca ${ }^{26}$.

Le 29 mars, seulement $42 \%$ des infirmières en France avaient reçu une dose de vaccin, contre $80 \%$ des médecins généralistes et $12 \%$ de la population général ${ }^{27}$. Les chiffres n'ont pas été moins choquants à la mi-juin, lorsque $L e$ Monde a eu accès à un document de l'Assistance PubliqueHôpitaux de Paris suggérant que si quatre-vingt-onze pour cent des médecins généralistes avaient reçu une dose, le personnel non médical, y compris les infirmières et les aides-soignantes, était encore loin derrière avec cinquante-quatre pour $\operatorname{cent}^{28}$. Il était toujours peu probable que ce chiffre augmente rapidement, car la stratégie européenne de commandes groupées a entraîné une pénurie de vaccins ${ }^{29}$. La décision d'utiliser l'âge comme seuil pour les infirmières, ainsi que pour le grand public, n'a pas arrangé les choses. L'utilisation du risque d'exposition virale aurait constitué une stratégie plus logique en ce qui concerne les infirmières. Toute personne travaillant aux soins intensifs aurait dû être la première à être vaccinée, quel que soit son âge. Lors de la première vague, la contamination était directement liée à l'exposition. Au-delà, le véritable problème est qu'une grande partie des contaminations résultent d'une transmission communautaire. En effet, la plupart des personnels hospitaliers se contaminant en dehors de l'hôpital, lors de rencontres privées $^{30}$.

Accélérer la campagne de vaccination, c'est faire en sorte que les vaccinations se fassent rapidement dans l'espoir que les hospitalisations pour COVID-19 diminuent. Israël en est un modèle. Le seuil symbolique de 4000 patients en soins intensifs a été franchi en France le 15 mars, dépassant le pic de la deuxième vague. Ce n'est que le 21 mai que ce chiffre est repassé sous la barre des 4000 patients ${ }^{31}$. La décision de suspendre AstraZeneca signifie que les hôpitaux ont été contraints de jeter des doses de vaccins prêtes à l'emploi et d'annuler des rendez-vous de vaccination $^{32}$. D'autre part, les médecins ont été invités à extraire une septième dose de Pfizer de flacons initialement prévus pour cinq doses ${ }^{33}$.
Les taux d'infection et les admissions en soins intensifs restant élevés, il est clair que la France et l'Europe doivent continuer à accélérer la campagne de vaccination. Plus tôt nous vaccinerons, plus tôt les éventuelles hésitations seront levées. Arrêter de vacciner pendant quarante-huit à soixante-douze heures au nom du principe de précaution, c'était reprendre à zéro le travail de sensibilisation aux vaccins. Toute décision prise doit tenir compte de la balance bénéfice-risque qui est largement en faveur de tous les vaccins disponibles à ce jour. Leur efficacité, comme l'ont montré les recherches menées tant en Europe qu'aux Etats-Unis, est, par définition, supérieure à cinquante pour cent $^{34}$. En conséquence, le vaccin d'AstraZeneca a été stigmatisé et des patients qui ne savent rien du plus simple calcul risque-bénéfice réclament maintenant le droit de choisir leurs vaccins ${ }^{35}$.

Au début de la pandémie, les masques ont d'abord été déclarés sans utilité, puis rendus obligatoires à l'intérieur, puis à l'extérieur, mais seulement à l'été 2020. Lorsque la première vague a balayé l'Europe au début de l'année 2020, la France a effectué moins de tests COVID que la plupart des autres pays d'Europe ${ }^{36}$, pour finalement dépister gratuitement toutes les personnes qui le souhaitaient, $y$ compris celles qui étaient asymptomatiques et sans ordonnance, en septembre $2020^{37}$.

Il est toujours facile de voir le verre à moitié vide plutôt qu'à moitié plein, mais peut-être aurait-il fallu appliquer dès le départ une décision à l'échelle européenne. Le principe de précaution aurait pu suggérer que, compte tenu du risque réel de maladie et de décès, toute l'Europe aurait dû être placée en quarantaine stricte. Si cette stratégie est bien connue comme étant une stratégie médiévale, elle n'en est pas moins efficace pour autant. Quel que soit le virus, soit il meurt avec son hôte, soit son hôte survit au virus ; s'il ne peut se propager, il ne peut survivre. Si une quarantaine complète et efficace est économiquement irréalisable, la confusion créée par la débâcle d'AstraZeneca était inacceptable dans une crise de santé publique ; le gouvernement français déclarant à la fois que le vaccin d'AstraZeneca est sûr compte tenu de la balance bénéfices-risques du vaccin, puis, 48 heures plus tard, déclarant que, puisque l'Allemagne a appliqué le principe de précaution, nous l'appliquerons aussi. Le vaccin d'AstraZeneca est désormais entaché, et restera un vaccin de seconde zone aux yeux du public, ne serait-ce que parce que les Français, au moins, se souviennent que des questions ont été soulevées quant à son efficacité et à ses effets indésirables potentiels ${ }^{38}$.

Il s'agit ici l'exemple probablement que la confusion nait souvent d'un défaut de communication. Les effets possibles du vaccin n'ont peut-être pas été suffisamment expliqués ; et personne au gouvernement n'a clairement fait la distinction entre risque individuel et bien-être collectif. Il faut garder en tête que le risque de thrombose provoqué par le vaccin AstraZeneca est inférieur aux 
risques de thrombose observés chez les jeunes fumeurs prenant des pilules contraceptives ostroprogestatives ${ }^{39}$. De même, les patients souffrant d'une infection au COVID19 courent un risque absolument clair et significativement accru d'événements thromboemboliques ${ }^{40}$. L'évaluation coût-bénéfice est entièrement en faveur de la vaccination par AstraZeneca. Une récente analyse de données effectuée par une équipe d'Oxford montre que le risque de développer une thrombose dans les deux semaines suivant un diagnostic de COVID-19 est de 39 sur 1 million $^{41}$. Dans les quinze jours suivant une piqûre de Moderna ou de Pfizer, la même étude a montré que ce chiffre tombe à quatre sur un million, tandis que l'estimation actuelle de l'EMA maintient les chances de thrombose de la piqûre d'AstraZeneca à un niveau légèrement moins favorable de cinq sur un million. La balance bénéfices-risques penche fortement en faveur de la vaccination.

Ce qui est nouveau et spécifique à cette épidémie, c'est l'association des noms des vaccins et des laboratoires. De plus en plus de personnes appellent les centres de vaccination pour demander le nom du vaccin qu'on va leur injecter. La géographie du monde est maintenant en cause, des vaccins apparaissant d'Amérique, d'Angleterre, de Russie et de Chine. A cela, s'ajoute le coût unitaire du vaccin. Les vaccins Pfizer et Moderna sont les plus chers, avec respectivement dix-neuf dollars et cinquante cents et quinze dollars par injection ${ }^{42}$. Il n'était pas dans l'intérêt de la France, ni de l'Europe, de suspendre la vaccination par AstraZeneca, un vaccin relativement bon marché. Sept millions de doses du vaccin Janssen de Johnson \& Johnson et trente-cinq millions de doses Pfizer doivent arriver en France d'ici la fin de l'été. Il n'y a pas le temps d'attendre qu'un vaccin soit remplacé par un autre de qualité équivalente, et c'est une vraie préoccupation. Finalement, c'est le contraire de ce que la France a vécu en 2009 avec la pandémie H1N1, lorsque Roselyne Bachelot, ministre de la Santé de l'époque, a ordonné le double de vaccins requis en négligeant de suivre les conseils actualisés selon lesquels les doses de rappel n'étaient pas nécessaires ${ }^{43}$. Heureusement, la pandémie de H1N1 n'a pas frappé l'Europe comme prévu, et cette erreur n'a pas influencé l'opinion publique.

Que faut-il faire maintenant, et à l'avenir, lorsque la prochaine pandémie se présentera. Nous avons besoin de quelque chose au-delà des publicités sanitaires actuelles dans lesquelles une grand-mère embrasse ses petits-enfants nettement peu enthousiastes parce qu'elle a été vaccinée. Pour briser la chaîne de transmission, il ne suffit pas de s'occuper des personnes âgées. En effet, Israël a commencé à vacciner les jeunes de seize à dix-huit ans à la fin du mois de janvier $2021^{44}$, et à la mi-mai, a commencé à préparer la vaccination des jeunes de douze à quinze ans ${ }^{45}$. Il faut avant tout redécouvrir les principes de décision qui ont, au moins, un contenu clair. On peut notamment citer l'exemple d'un bénéfice sanitaire collectif qui au-delà de la levée des restrictions permet aux individus un certain retour à une vie d'avant comme avec le passeport sanitaire. Ce qui est déterminant dans la promotion d'un bénéfice collectif n'est pas exclusivement l'efficacité d'un vaccin à protéger les formes symptomatiques d'une maladie, mais d'éliminer le virus en premier lieu. Cela ne peut se faire sur la base d'une quelconque évaluation individuelle des risques.

Une dernière mise en garde s'impose peut-être. L'humanité a eu de la chance avec le COVID-19. Certaines maladies sont bien plus mortelles, mais se propagent lentement. Bien que le COVID-19 se propage rapidement, il n'est pas terriblement mortel.

La prochaine pandémie pourrait être les deux.

Benjamin Davido est Infectiologue et directeur de la médecine de crise pour l'épidémie de covid-19 à l'hôpital Raymond-Poincaré de Garches.

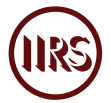

1. «Une augmentation de $65 \%$ de SEP après le pic de vaccination contre l'hépatite B en 1994 », Le quotidien du medecin, 1 Décembre, 2014.

2. "U.S. and U.K. Are Optimistic Indicators for COVID-19 Vaccination Uptake (Les États-Unis et le Royaume-Uni sont des indicateurs optimistes pour l'adoption de la vaccination par le COVID-19) », Ipsos, 29 Décembre, 2020.

3. Kyle Blankenship, " AstraZeneca Hopes Warmer Storage Needs for COVID-19 Vaccine Will Be an Advantage against mRNA Competitors (AstraZeneca espère que les besoins de stockage plus chauds du vaccin COVID-19 constitueront un avantage par rapport aux concurrents à ARNm) ", Fierce Pharma, 23 Novembre, 2020.

4. Minnesota Department of Health, Interim COVID-19 Vaccine Provider Guide, Appendix A: Pfizer-BioNTech COVID-19 Vaccine (Guide provisoire du fournisseur du vaccin COVID19, Annexe A : Vaccin COVID-19 de Pfizer-BioNTech) (Minnesota : Minnesota Department of Health, 2021).

5. Agence européenne des médicaments, «EMA recommends COVID-19 Vaccine AstraZeneca for authorisation in the EU (L'EMA recommande l'autorisation du vaccin COVID-19 d'AstraZeneca dans l'UE) », 29 Janvier, 2021.

6. «Macron: AstraZeneca vaccine 'quasi-ineffective' for over- 65 s (Macron : le vaccin d'AstraZeneca est « quasi-inefficace » pour les personnes âgées de plus de 65 ans.) », France 24, 29 Janvier, 2021.

7. « Stratégie de vaccination contre la Covid-19 - Place du Covid-19 Vaccine AstraZeneca », Haute Autorité de santé, 2 Février, 2021.

8. «AstraZeneca : la HAS recommande son utilisation chez les professionnels de santé et les personnes de 50 à 64 ans ", Haute Autorité de santé, 2 Février, 2021. 
9. Thomas Dubois, "Effets secondaires du vaccin AstraZeneca : 'Un moment un peu désagréable' ", Paris Normandie, 12 Février, 2021.

10. Viviane Le Guen, « Coronavirus : pourquoi les soignants sontils divisés sur la vaccination? » France Bleu, 5 Mars, 2021.

11. " COVID-19 Vaccine AstraZeneca: Benefits Still Outweigh the Risks Despite Possible Link to Rare Blood Clots with Low Blood Platelets (Vaccin COVID-19 AstraZeneca : Les avantages l'emportent toujours sur les risques malgré un lien possible avec de rares caillots sanguins en cas de faibles plaquettes sanguines) ", European Medicines Agency, 18 Mars, 2021.

12. Jacqui Wise, " Covid-19: European Countries Suspend Use of Oxford-AstraZeneca Vaccine After Reports of Blood Clots (COVID-19: Les pays européens suspendent l'utilisation du vaccin d'Oxford-AstraZeneca après des rapports de caillots sanguins) », BMJ, 11 Mars, 2021, doi : 10.1136/bmj.n699. Les mêmes chiffres ont été cités dans un article publié dans The Lancet à la fin du mois : «Thromboembolism and the OxfordAstraZeneca COVID-19 Vaccine: Side-Effect or Coincidence? (Thromboembolie et le vaccin COVID-19 d'Oxford-AstraZeneca : Effet secondaire ou coïncidence ?) ", The Lancet 397, no. 10,283 (2021) : 1,441-43, doi : 10.1016/S0140-6736(21) 00762-5.

13. "Germany Suspends Use of AstraZeneca Vaccine, Along with Italy, France, Spain (L'Allemagne suspend l'utilisation du vaccin d'AstraZeneca, tout comme l'Italie, la France et l'Espagne) », $D W, 15$ March, 2020.

14. Sarah Wheaton, « Revenge of the Precautionary Principle (La revanche du principe de précaution) », Politico, 18 Mars, 2021.

15. "EMA Press Conference (Conférence de Presse de l'EMA)», European Medicines Agency (Vidéo YouTube), 18 Mars, 2021. Le segment pertinent commence à 41:57.

16. " Principle of Precaution Must Prevail, says EU Ambassador (Le principe de précaution doit prévaloir, déclare l'ambassadeur de l'UE) », BBC, 16 Mars, 2021 ; Wheaton, « Revenge of the Precautionary Principle ".

17. «WHO says countries should keep Using AstraZeneca COVID Vaccine as European Countries Suspend Use (L'OMS déclare que les pays doivent continuer à utiliser le vaccin COVID d'AstraZeneca alors que les pays européens suspendent son utilisation) », EuroNews, 16 Mars, 2021.

18. " Principe de Précaution ", la Commission Européenne, 2 Février, 2000.

19. Per Sandin, "Dimensions of the Precautionary Principle (Dimensions du principe de précaution) », Human and Ecological Risk Assessment: An International Journal 5, no. 5 (1999) : 889-907, doi : 10.1080/10807039991289185.

20. " Principe de Précaution ", la Commission Européenne.

21. Gary Marchant et Kenneth Mossman, Arbitrary and Capricious The Precautionary Principle in the European Union Courts (Londres : International Policy Press, 2005), 25.

22. "Communication de la Commission sur le recours au principe de précaution », Communiqué de la Commission Européenne, 2 Février, 2000.
23. "Communication de la Commission ", la Commission Européenne.

24. Merryn Voysey et al., "Single Dose Administration, and the Influence of the Timing of the Booster Dose on Immunogenicity and Efficacy of ChAdOxI nCoV-19 (AZD1222) Vaccine (Administration d'une dose unique et influence du moment de l'administration de la dose de rappel sur l'immunogénicité et l'efficacité du vaccin ChAdOx1 nCoV-19 (AZD1222)) », The Lancet (pre-print) (2021), doi : 10.2139/ssrn.3777268.

25. " Covid-19: le vaccin AstraZeneca n'est 'pas le plus adapté' pour 'une population aussi exposée que les soignants', estime un syndicat d'infirmiers ", Franceinfo, 5 Mars, 2021.

26. Olivier Véran, Publication sur Twitter, 5 Mars, 2021, 17 h30.

27. « Point Épidémiologique COVID-19 no. 59, 15 avril, 2021 ", Santé Publique France, 54.

28. Stéphane Mandard et Camille Stromboni, " Covid-19: pourquoi la vaccination plafonne chez les infirmiers et les aides-soignants ", Le Monde, 18 Juin, 2021.

29. Ben Hall et al., " Shortage of Coronavirus Shots Heaps Pressure on European Leaders (La pénurie de vaccins contre le coronavirus met les dirigeants européens sous pression) ", Financial Times, 29 Janvier, 2021.

30. Benjamin Davido et al., " The First Wave of COVID-19 in Hospital Staff Members of a Tertiary Care Hospital in the Greater Paris Area: A Surveillance and Risk Factors Study (La première vague de COVID-19 chez les personnels hospitaliers d'un hôpital tertiaire de la région parisienne : Une étude de surveillance et de facteurs de risque) », International Journal of Infectious Diseases 105 (2021) : 172-79, doi : 10.1016/j.ijid.2021.02.055.

31. Hannah Ritchie et al., " Coronavirus Pandemic (COVID19) - the Data (Pandémie de coronavirus (COVID-19) - les données) », Our World in Data (2021).

32. Académie Nationale de Médicine, "Entre l'indispensable précaution et l'indésirable confusion », 23 Mars, 2021.

33. Klervi Drouglazet, « Covid-19. Est-il vraiment possible d'extraire une septième dose des flacons du vaccin Pfizer?", Ouest France, 13 Mars, 2021.

34. Jamie Lopez Bernal et al., «Effectiveness of the Pfizer-BioNTech and Oxford-AstraZeneca Vaccines on Covid-19 Related Symptoms, Hospital Admissions, and Mortality in Older Adults in England: Test Negative Case-Control Study (Efficacité des vaccins Pfizer-BioNTech et Oxford-AstraZeneca sur les symptômes liés au Covid-19, les admissions à l'hôpital et la mortalité chez les adultes plus âgés en Angleterre : Étude cas-témoins negative) », The British Medical Journal 373, no. 1,088 (2021), doi : 10.1136/bmj.n1088.

35. Par exemple, Huguette Poitras, " Choisir son vaccine ", Le Devoir, Mars 22, 2021 : «Plusieurs voix s'élèvent en ce moment pour revendiquer le droit de choisir son vaccin contre la COVID-19».

36. Louis Tanca, « La France teste beaucoup moins sa population que les autres ", BFMTV, 31 Mars, 2020.

37. " Tests PCR : plus besoin d'une ordonnance pour en bénéficier et être remboursé », Direction de l'information 
légale et administrative (Premier ministre), 18 Septembre, 2020.

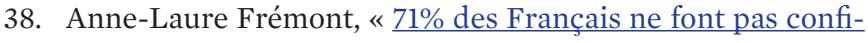
ance au vaccin AstraZeneca », Le Figaro, 8 Avril, 2021.

39. " Birth Control Creates Higher Risk of Blood Clots than AstraZeneca Vaccine (Les pilules contraceptives œstroprogestatives présentent un risque plus élevé de caillots sanguins que le vaccin d'AstraZeneca) », Open Access Government, 16 Mars, 2021.

40. Mahmoud Malas et al., " Thromboembolism Risk of COVID19 Is High and Associated with a Higher Risk of Mortality: A Systematic Review and Meta-Analysis (Le risque thromboembolique de COVID-19 est élevé et associé à un risque de mortalité plus élevé : Une revue systématique et une méta-analyse) », EClinicalMedicine, 29, no. 100639 (2020), doi : 10.1016/j.eclinm.2020.100639.

41. Maxime Taquet et al., "Cerebral Venous Thrombosis and Portal Vein Thrombosis: A Retrospective Cohort Study of 537,913 COVID-19 Cases (Thrombose veineuse cérébrale et thrombose de la veine portale : Une étude de cohorte rétrospective de 537913 cas COVID-19) », medRxiv, Mai 11,
2021, doi : 10.1101/2021.04.27.21256153. Pour un résumé, voir Shawna Williams, « Blood Clot Risk from COVID-19 Higher than After Vaccines: Study (Le risque de caillot sanguin lié à COVID-19 est plus élevé que celui lié aux vaccins : Étude) », The Scientist, 16 Avril, 2021.

42. Eric Sagonowsky, " Pfizer Eyes Higher Prices for COVID19 Vaccine after the Pandemic Wanes (Pfizer envisage une hausse des prix du vaccin COVID-19 après le déclin de la pandémie) », Fierce Pharma, 23 Février, 2021.

43. Sandrine Cabut, " Vaccins H1N1 : le ministère de la Santé mis en cause », Le Figaro, 14 Octobre, 2010.

44. "Israel Expands Vaccination Campaign to Teens Aged 16-18 (Israël étend la campagne de vaccination aux adolescents âgés de 16 à 18 ans) ", The Times of Israel, 23 Janvier, 2021.

45. «Israel Set to Vaccinate 12- to 15-Year-Olds after FDA Okays Pfizer Shot (Israël s'apprête à vacciner les jeunes de 12 à 15 ans après que la FDA a approuvé le vaccin de Pfizer) », The Times of Israel, 11 May, 2021.

DOI: $10.37282 / 991819.21 .25$ 